\title{
La experiencia de la UNED en el marco de las capacitaciones de inglés al MEP
}

\author{
Yinnia Mora Ordóñez*
}

\author{
Recibido: Marzo 2012 • Aceptado: Mayo 2012
}

\begin{abstract}
RESUMEN
El presente artículo contiene el análisis de los resultados de la capacitación de Inglés de las zonas atendidas por la UNED, dentro del marco del Proyecto de Capacitación a Docentes de Inglés en Servicio del Ministerio de Educación Pública (MEP) durante el periodo comprendido entre los años 2008 y 2011. El proyecto fue coordinado y ejecutado por la Comisión Técnica- Académica MEP CONARE, conformada para ese fin por dos representantes de cada una de las universidades públicas del Consejo Nacional de Rectores (CONARE) y por una representante del Instituto de Desarrollo Profesional (IDP) Uladislao Gámez del Ministerio de Educación Pública.
\end{abstract}

Palabras clave: capacitaciones, inglés, dominio lingüístico, UNED, MEP.

\begin{abstract}
This article presents the analysis of the results obtained in the English training project conducted by UNED and the other three public Universities along with the Ministry of Education of Costa Rica. The analysis takes into account the results of the Regional areas trained by UNED during the years 2008 through 2011. The name of the project is "Training for English educators in service for MEP". It was coordinated and implemented by a group of academics from the four public Universities of Costa Rica and an advisor from MEP.
\end{abstract}

Key words: training, English, linguistic competence, UNED, MEP.

\section{Antecedentes}

Gracias a la integración de Costa Rica al mundo globalizado y su inserción en mercados internacionales se han favorecido las condiciones de una progresiva demanda de recurso humano con

* Bachiller en Inglés de la UCR, licenciada en Docencia con énfasis en Enseñanza del Inglés de la UNED, máster en Administración Educativa de la UNED. Encargada de la carrera de Enseñanza del Inglés para I y II Ciclos de la UNED; coordinadora por parte de la UNED del Proyecto de Capacitaciones de Inglés MEP-CONARE. 
dominio del idioma inglés, tanto por parte del sector productivo nacional y transnacional, como por parte del sector académico. El acceso al conocimiento mundial conduce al país a plantearse diversos retos, uno de ellos, el desarrollo de las competencias lingüísticas en inglés. Dominar este idioma, posicionado como el segundo más hablado en el mundo, es una prioridad en el mercado laboral.

Aunque los esfuerzos realizados en Costa Rica a finales del siglo XX para impulsar el aprendizaje del inglés resultaron valiosos -este se incluyó en las escuelas públicas apenas en 1994- es innegable que si se deseaba seguir mejorando la habilidad de los(as) estudiantes para comunicarse de manera fluida en dicho idioma, se evidenciaba un impulso mayor y mucho más sistemático. Así, en el año 2008, el gobierno de Costa Rica decretó la creación de la Iniciativa Costa Rica Multilingüe, que pretende "el desarrollo de competencias lingüísticas que permitan a los costarricenses aprovechar las oportunidades laborales que el mundo globalizado presenta" (Decreto 34 425-MEPCOMEX, 11 marzo 2008). A partir de este decreto, uno de los ejes fundamentales que desarrolla el MEP es un proyecto de capacitación para mejorar la calidad del recurso humano con formación bilingüe en el idioma inglés.
Este proyecto responde, específicamente, a las necesidades de capacitación en dominio lingüístico del inglés de la población docente analizadas en dos foros de discusión, a saber, el Primer Foro Nacional de Inglés y el Plan Nacional de Inglés. El Primer Foro Nacional de Inglés, realizado el 30 de noviembre de 2005, contó con la participación de representantes del Consejo Nacional de Rectores (CONARE), el Ministerio de Educación Pública (MEP), la Oficina de Planificación de la Educación Superior (OPES) y la Coalición Costarricense de Iniciativas de Desarrollo (CINDE), entre otros. En él se analizaron la importancia del inglés para el desarrollo económico del país y las formas de mejorar el dominio de este idioma por parte de la población costarricense. Algunas de las conclusiones y medidas planteadas fueron las siguientes:

\section{Conclusiones}

"a. Los programas del MEP limitan al docente y no se enfocan en las metodologías de enseñanza más apropiadas y eficaces.

b. La macroevaluación de inglés del MEP no mide la destreza oral efectivamente y limita a los profesores a la enseñanza de lectura únicamente, dejando de lado las otras tres 
destrezas [comprensión auditiva, producción oral $\mathrm{y}$ producción escrita].

c. La habilidad comunicativa de la población docente de inglés debe mejorarse para alcanzar un nivel intermedio alto." (Cámara Costarricense de Tecnologías de Información y Comunicación, 2005: 1)

\section{Medidas}

"a Creación de alianzas estratégicas entre el MEP y el CONARE.

b. Programas de capacitación y actualización profesional permanentes y obligatorios." (Cámara Costarricense de Tecnologías de Información y Comunicación, 2005: 8)

Por otra parte, el Plan Nacional de Inglés fue elaborado por un grupo interinstitucional constituido por autoridades del MEP, Ministerio del Comercio Exterior (COMEX), Ministerio de Economía, Industria y Comercio (MEIC), Instituto Nacional de Aprendizaje (INA), CINDE y Estrategia Siglo XXI.

Las acciones primordiales concretas del Plan, pertinentes para este proyecto, son las siguientes:

- "Desarrollo de un programa masivo de formación y capacitación de profesores de inglés". (MEP, COMEX, MEIC, INA, CINDE \& Estrategia Siglo XXI, 2007: 33)

- "Capacitación en el uso de metodologías innovadoras en la enseñanza del inglés, acorde con las necesidades de la Costa Rica del siglo XXI". (MEP y otros, 2007: 39)

El MEP, como ente ejecutor, planteó acciones concretas para mejorar los procesos de enseñanza y aprendizaje a partir de tres ejes: a) diagnóstico y capacitación docente, b) revisión y diseño de todos los programas de estudio en todos los niveles y modalidades, c) infraestructura física y tecnológica (Cámara Costarricense de Tecnologías de Información y Comunicación, 2005).

Durante el periodo entre marzo y junio de 2008 y como parte del eje de diagnóstico y capacitación docente, se administró una prueba internacional a la población de docentes de Inglés en servicio que laboraban en el MEP, la cual permitió conocer su nivel de dominio lingüístico. Este diagnóstico se realizó a nivel nacional mediante la administración del Test of English for International Communication (TOEIC), cuyos resultados fueron equiparados con los descriptores del Marco Común Europeo de Referencia para las Lenguas Vivas. 
El MEP administró esta prueba estandarizada a un total de 3 193 docentes de Inglés de todo el país para medir su dominio lingüístico. El número de docentes que realizó la prueba representa aproximadamente un $86 \%$ de la población docente del país convocada. Los resultados de la prueba TOEIC aplicada en el 2008 son los siguientes:

CUADRO 1

Resultados de la prueba TOEIC aplicada en el 2008

\begin{tabular}{lc} 
Nivel de dominio lingüístico & Número de docentes \\
A1 (usuario básico elemental) & 232 \\
A2 (usuario básico) & 980 \\
B1 (usuario independiente intermedio bajo) & 905 \\
B2 (usuario independiente intermedio) & 645 \\
C1 (usuario competente) & 431 \\
\hline
\end{tabular}

Fuente: Divulgación de resultados realizada por el señor Ministro de Educación, Dr. Leonardo Garnier Rímolo, en conferencia de prensa del 6 de agosto de 2008.

El sector de la población con dominio básico representa un $37,96 \%$, mientras que el correspondiente a dominio intermedio representa un $48,54 \%$. La población con dominio intermedio alto corresponde al $14 \%$.

El Consejo Nacional de Rectores (CONARE), entidad constituida por las cuatro universidades públicas, a saber, Universidad Nacional, Universidad de Costa Rica, Universidad Estatal a Distancia e Instituto Tecnológico de Costa Rica, como ente ejecutor en el Plan Nacional, aceptó el reto de apoyar al MEP en una capacitación a nivel nacional dirigida a los(as) docentes de Inglés tomando como base el diagnóstico antes citado. Para esto suscribieron el Convenio Marco de Cooperación el 23 de junio de 2008, cuyo objetivo es:

"establecer un instrumento eficaz de cooperación y coordinación con las instituciones de educación superior para el desarrollo de programas y actividades conjuntas que promuevan el aumento de la capacidad profesional y el desarrollo humano del personal en servicio del Ministerio de Educación Pública, en áreas de común interés, que contempla, entre otros, la ejecución de actividades de desarrollo profesional, dirigidas al personal en servicio del sistema educativo." (MEP-CONARE, 2008: 2) 
A partir de ese convenio de cooperación, CONARE, en coordinación con el MEP, diseñó y ejecutó el proyecto de capacitación para docentes de Inglés en servicio denominado MEP-CONARE Inglés.

\section{Gestión del proyecto}

Con base en los convenios específicos entre el Ministerio de Educación Pública y el Consejo Nacional de Rectores firmados entre los años 2008 y 2011, las universidades asumieron la responsabilidad de capacitar docentes de las 27 direcciones regionales de enseñanza del MEP.

Para la distribución de las distintas direcciones regionales de enseñanza del país, se tomó en cuenta la infraestructura que tiene cada universidad en dichas zonas. En los años 2009 y 2010 el MEP amplió el número de direcciones regionales de enseñanza y pasó de 21 a 27; las nuevas regiones se asignaron según la zona geográfica cubierta por cada universidad. La UNED se responsabilizó para realizar esta tarea en ocho direcciones regionales de enseñanza: San José Norte, San José Este, San José Oeste, Desamparados, Puntarenas, Peninsular, Cañas y Coto.

El objetivo general planteado por el MEP y CONARE para este proyecto fue "capacitar en el área de dominio lingüístico y en metodologías para la enseñanza a la población docente de inglés que labora en el sector público de educación preescolar, primaria y secundaria del país" (Convenio Específico MEP CONARE, 2011: 3)

\section{Metodología}

La Comisión MEP-CONARE tuvo la responsabilidad de planificar, diseñar, organizar, ejecutar y evaluar las capacitaciones en las 27 direcciones regionales de enseñanza del MEP. A su vez, a la Comisión le correspondió tomar todas las decisiones académicas y administrativas respecto a la creación de programas de curso, evaluaciones, actividades de evaluación, libro de texto a utilizar, contratación de capacitadores/as, entre otras labores pertinentes.

El proyecto tuvo dos ejes fundamentales de capacitación: dominio lingüístico del inglés y destrezas metodológicas para la enseñanza del idioma con poblaciones de docentes de preescolar, primaria y secundaria. En este artículo se presentan los resultados obtenidos en los cursos de dominio lingüístico.

La primera etapa contempló la capacitación en cursos de dominio lingüístico con énfasis en las destrezas de comprensión auditiva y producción oral. Se ofertaron nueve cursos con una duración en promedio de 90 horas. La población meta 
era de docentes que poseían nivel de dominio A2 y A1. En el 2009 y 2010 se unieron a la capacitación los docentes con nivel B1. Cada docente se capacitó un promedio de 500 horas (dependiendo de la ubicación en la banda) antes de volver a tomar una prueba que midiera su nivel de dominio lingüístico.

La segunda etapa se inició en el 2010 y contempló cuatro cursos de 80 horas en promedio. Se cubrieron las áreas de métodos y técnicas para la enseñanza, elaboración de materiales, adecuaciones curriculares y evaluación. La población meta de esta etapa de la capacitación poseía un nivel de dominio lingüístico $\mathrm{B} 2 \mathrm{y} \mathrm{C} 1$.

\section{Población beneficiada directa}

El total de la población capacitada a través de este proyecto fue de 3285 docentes de Inglés de preescolar, primaria y secundaria en servicio. De esa población, a la UNED le correspondió capacitar un promedio aproximado de 800 docentes de Inglés de las direcciones regionales de enseñanza del Ministerio de Educación Pública en San José Norte, San José Este, San José Oeste, Desamparados, Puntarenas, Coto y Cañas. El cuadro 2 detalla el número de personas capacitadas a nivel nacional así como el total correspondiente a la UNED.

CUADRO 2

Población beneficiada

\begin{tabular}{lcccc} 
& Año 2008 & Año 2009 & Año 2010 & Año 2011 \\
Población atendida por UNED & 294 & 454 & 977 & 663 \\
Población total Proyecto MEP-CONARE & 1093 & 1879 & 3910 & 3285 \\
\hline
\end{tabular}

Fuente: Formularios FOCAP 6 2008-2011.

\section{Población beneficiada indirecta}

Está compuesta por estudiantes de preescolar, primaria y secundaria de las cinco direcciones regionales de enseñanza del Ministerio de Educación Pública, a saber, San José Norte, San José Este, San
José Oeste, Desamparados, Puntarenas, Coto y Cañas, que le correspondió capacitar a la UNED. Estas poblaciones estudiantiles están expuestas al idioma inglés bajo la guía de docentes con mayor dominio lingüístico y mejores prácticas en mediación pedagógica. 


\section{Impacto del proyecto}

Este proyecto ha tenido un impacto directo en la población de docentes de Inglés de prescolar, primaria y secundaria del sistema educativo público, quienes han tenido la oportunidad de mejorar destrezas tanto en el área lingüística como metodológica. El impacto indirecto de este proyecto se verá en un mejor manejo del idioma inglés por parte de la población estudiantil arriba mencionada así como una mejor metodología para su enseñanza.

\section{Beneficio obtenido}

Como producto de esta capacitación, el país cuenta con docentes de Inglés con un dominio lingüístico, en su mayoría, de usuario independiente, el cual les permite impartir mejores lecciones en el idioma meta y preparar a sus estudiantes para ser más competitivos al enfrentar los retos laborales futuros.

\section{Beneficios recibidos por la UNED con el proyecto}

Tanto el MEP como las universidades se han visto favorecidas en gran medida con este proyecto de capacitaciones. Algunos beneficios que la UNED ha obtenido son:
- Fortalecimiento de su imagen como institución.

- Fortalecimiento de alianzas estratégicas entre CONARE y el Ministerio de Educación.

- Apertura de los(as) profesionales universitarios(as) hacia la comunidad de docentes de Inglés de prescolar, primaria y secundaria de la educación pública del país.

- Nuevos proyectos en áreas prioritarias para la mejora y fortalecimiento de la educación pública que redunden en una mayor competitividad del país.

- Creación de un reglamento para la administración de proyectos con fondos externos.

- Insumos, para la carrera de Enseñanza del Inglés, en relación con las fortalezas y debilidades en la práctica docente.

\section{Evaluación del proyecto}

El proyecto fue evaluado de manera sistemática utilizando cuestionarios dirigidos a los docentes participantes e instrumentos de observación de clase, los cuales fueron elaborados por la Comisión. Así mismo, se tabuló y sistematizó la información contenida en los FOCAP (formularios de capacitación), requeridos por el Instituto de Desarrollo Profesional. Los 
resultados de estas evaluaciones se presentaron en los respectivos informes anuales entregados al MEP y a la Vicerrectoría Académica.

Otras formas de evaluación continua fueron las siguientes:

1. Reuniones semanales o quincenales de la Comisión Técnica-Académica, según las necesidades del proyecto, para evaluar los materiales, los tipos de actividades, los instrumentos de evaluación y las decisiones técnico-administrativas, entre otros.
2. Comunicación, por parte de las coordinadoras con los/las capacitadores/as mediante reuniones, llamadas telefónicas y correos electrónicos para informar sobre lineamientos por seguir, revisar los exámenes y quices elaborados y pedir informes sobre el rendimiento de los/as participantes.

3. Informes periódicos enviados por los(as) capacitadores(as) a la Coordinación de este proyecto.

CUADRO 3

Inversión económica del 2008 al 2011

\begin{tabular}{|c|c|c|c|c|}
\hline & Año 2008 & Año 2009 & Año 2010 & Año 2011 \\
\hline Inversión total del MEP & $£ 300000000,00$ & c780000 000,00 & $\varnothing 625000000,00$ & 0,00 \\
\hline Presupuesto UNED & e78 535146,52 & ¿185486694,00 & c 156814584,00 & c151381 776,00 \\
\hline
\end{tabular}

Fuente: Convenios Específicos 2008-2011, Presupuestos UNED 2008-2011.

\section{Inversión}

El Ministerio de Educación Pública realizó una inversión aproximada de $\$ 2200000000$ (dos mil doscientos millones de colones), de los cuales la UNED ejecutó ф572218200 (quinientos setenta y dos millones doscientos dieciocho mil doscientos colones). En el cuadro 3 se detalla la inversión por año.

\section{Oferta de cursos de capacitación en dominio lingüístico}

Del periodo 2008 al 2011 se ofrecieron nueve cursos de capacitación en el área de dominio lingüístico dirigidos a docentes de Inglés de las 27 direcciones regionales del país. Los nombres de los cursos, las características y población beneficiada se presentan en el siguiente cuadro: 
CUADRO 4

Cursos de capacitación de dominio lingüístico

Nombre del curso
Características del curso

2008

MCE A1 Destrezas Lingüísticas para Docentes de Inglés en Servicio

MCE A2 Destrezas Lingüísticas para Docentes de Inglés en Servicio

2009

MCE B1- Destrezas Lingüísticas para Docentes de Inglés en Servicio

MCE B1+ Destrezas Lingüísticas para Docentes de Inglés en Servicio

2010

Estrategias de Comprensión Auditiva, Comprensión de Lectura y Expresión Oral

Comprensión Auditiva y Expresión Oral con Fines

Académicos I para Docentes de Inglés en Servicio

2011

Técnicas de Lectura y Expresión Oral I para Docentes de Inglés en Servicio

Comprensión Auditiva y Expresión Oral con Fines Académicos II para Docentes de Inglés en Servicio

Técnicas de Lectura y Expresión Oral II para Docentes de Inglés en Servicio
10 horas por semana

para un total de 140 horas

10 horas por semana

para un total de 140 horas

6 horas por semana

para un total de 102 horas

6 horas por semana

para un total de 102 horas

10 horas por semana

para un total de 70 horas

6 horas por semana

para un total de 120 horas

96 horas en total, una sesión

semanal de 6 horas por 16 semanas

120 horas en total, una sesión semanal de 6 horas por 15 semanas y 30 horas en línea

90 horas en total, una sesión semanal de 6 horas por 15 semanas

Fuente: Elaboración propia.

\section{Presentación y análisis de datos}

En el siguiente apartado se describen y analizan las zonas atendidas por la UNED, con base en los datos obtenidos en la capacitación a docentes de Inglés del MEP comprendida entre los años 2008 al 2011. Se presenta la cantidad de docentes capacitados y el rendimiento académico en cada uno de los cursos en las áreas de dominio lingüístico.

Finalmente, se presenta el panorama general de las regiones atendidas por la UNED. Se compara la ubicación del número de docentes que hicieron la prueba TOEIC en el 2008 con los que la hicieron en el 2011. Para efectos de facilitar la lectura del análisis de los gráficos se utilizará la siguiente nomenclatura: 
CUADRO 5

Nomenclatura utilizada en los gráficos para los cursos de dominio lingüístico

\begin{tabular}{|c|c|}
\hline \multicolumn{2}{|l|}{ Cursos Dominio Lingüístico } \\
\hline Nombre oficial del curso & $\begin{array}{l}\text { Nomenclatura } \\
\text { en los gráficos }\end{array}$ \\
\hline MCE A1 Destrezas Lingüísticas para Docentes de Inglés en Servicio & A1 \\
\hline MCE A2 Destrezas Lingüísticas para Docentes de Inglés en Servicio & A2 \\
\hline MCE B1- Destrezas Lingüísticas para Docentes de Inglés en Servicio & B1- \\
\hline MCE B1+ Destrezas Lingüísticas para Docentes de Inglés en Servicio & $\mathrm{B} 1+$ \\
\hline $\begin{array}{l}\text { Estrategias de Comprensión Auditiva, } \\
\text { Comprensión de Lectura y Expresión Oral }\end{array}$ & Estrategias \\
\hline $\begin{array}{l}\text { Comprensión Auditiva y Expresión Oral } \\
\text { con Fines Académicos I para Docentes de Inglés en Servicio }\end{array}$ & Comprensión I \\
\hline $\begin{array}{l}\text { Comprensión Auditiva y Expresión Oral } \\
\text { con Fines Académicos II para Docentes de Inglés en Servicio }\end{array}$ & Comprensión II \\
\hline $\begin{array}{l}\text { Técnicas de Lectura y Expresión Oral I } \\
\text { para Docentes de Inglés en Servicio }\end{array}$ & Técnicas I \\
\hline $\begin{array}{l}\text { Técnicas de Lectura y Expresión Oral II } \\
\text { para Docentes de Inglés en Servicio }\end{array}$ & Técnicas II \\
\hline
\end{tabular}

Fuente: Elaboración propia.

\section{Análisis de las regiones capacitadas por la UNED}

El gráfico 1 muestra la población capacitada de las regiones atendidas por la UNED en los nueve cursos de dominio lingüístico ofertados entre los años 2008 y 2011.

Se observa que el curso de nivel básico A1 se ofertó a 40 docentes en el año 2008. El curso A2, en el año 2008, se brindó a 189 y, en el año 2009, se ofreció nuevamente, esta vez a 70 participantes; en total se capacitaron 259 docentes en la banda $\mathrm{A} 2$ en los dos periodos que comprenden 2008 y 2009.
El curso B1- se ofreció en el año 2009 y participaron 528 docentes. Al curso B1+, ofertado dos años consecutivos, asistió un total de 484 participantes: 337 en el 2009 y 147 en el 2010.

El curso de Estrategias se ofreció en el 2010 y 2011 y lo llevó un total de 443 docentes.

El curso de Comprensión I se brindó en el 2010 y lo cursaron 79. En el 2011 se ofertó el curso de Comprensión II y en este participaron 62 docentes. Es importante anotar que el curso de Comprensión I fue requisito para el de Comprensión II. 


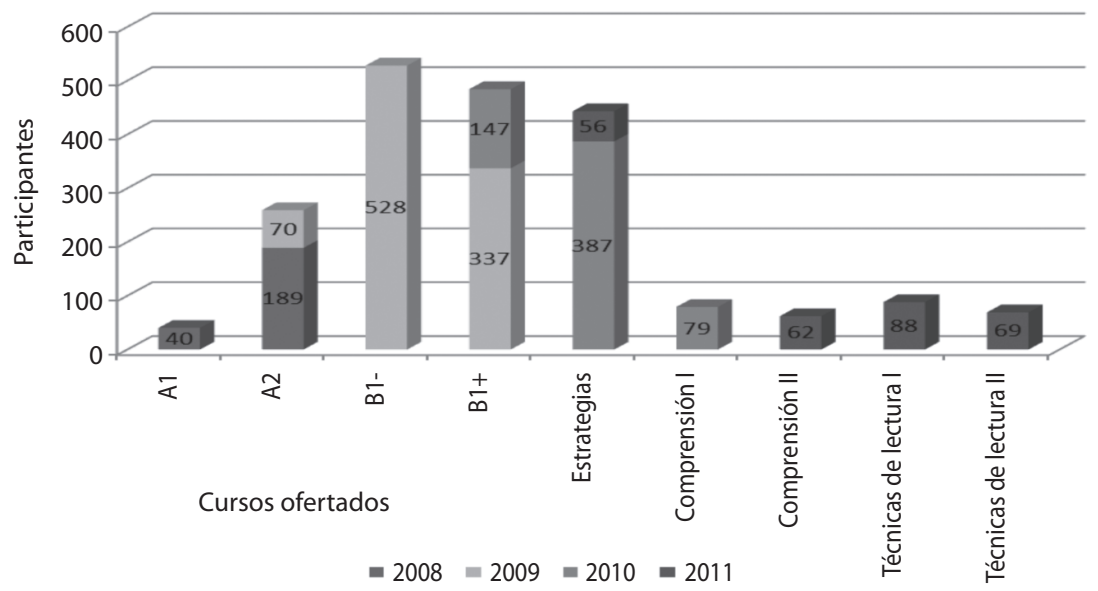

Gráfico 1. Cantidad de docentes capacitados(as) en dominio lingüístico según cursos ofertados entre 2008 y 2011, regiones capacitadas por la UNED. Fuente: Archivos FOCAP 6, Departamento Seguimiento y Evaluación.

Los cursos de Técnicas de Lectura I y II se ofrecieron en el 2011, y asistieron 88 y 69 docentes respectivamente. Cabe destacar que el curso Técnicas de Lectura I fue requisito para cursar el siguiente.

La información contenida en el gráfico 1 muestra que se capacitaron en los cursos de dominio lingüístico un total aproximado de 695 docentes de Inglés en las diferentes modalidades y niveles.

El gráfico 2 destaca las cifras de aprobación y reprobación del personal docente que participó en los nueve cursos de dominio lingǘstico ofertados entre los años 2008 y 2011 en las regiones capacitadas por la UNED.
El curso A1, ofrecido en el 2008, muestra resultados de aprobación de 33 docentes, y fue reprobado por siete. El curso A2 se ofertó dos veces, como se notó en el gráfico 1; por lo tanto, en el gráfico 2 se observa que, producto de las ofertas, lo aprobaron 247 docentes y lo reprobaron 43.

El curso B1- fue aprobado por 462 personas y lo reprobaron únicamente 66. Para el curso B1+ de 484 docentes que lo cursaron en total en los dos periodos en que se ofertó, 379 lo aprobaron y 105 lo reprobaron.

En los dos periodos en que se brindó el curso de Estrategias, de los 443 participantes, 375 lo aprobaron y 68 lo reprobaron. 


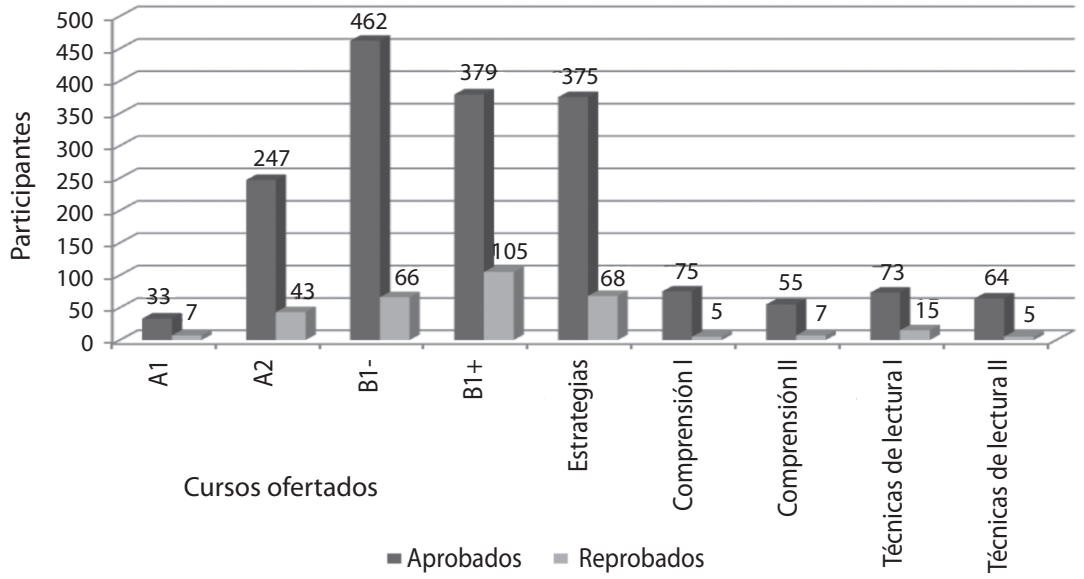

Gráfico 2. Rendimiento académico de los(as) docentes capacitados(as) en dominio lingüístico según cursos ofertados entre 2008 y 2011, regiones capacitadas por la UNED. Fuente: Archivos FOCAP 6, Departamento Seguimiento y Evaluación.

El curso de Comprensión I fue aprobado por 75 docentes y reprobado por cinco. En el de Comprensión II, la promoción fue de 55 docentes, y siete lo reprobaron.

Para el curso de Técnicas de Lectura I, se observa una aprobación de 73 docentes y lo perdieron 15. El de Técnicas II fue aprobado por 64 docentes y reprobado por 5 .

En promedio, el curso con mayor aprobación fue MCE A1, Destrezas Lingüísticas para Docentes de Inglés en Servicio, con una aprobación del 95,36\%. Por el contrario, el curso menos aprobado fue MCE B1-, Destrezas Lingüísticas para Docentes de Inglés en Servicio, con una aprobación del 78,30\%.

En el Gráfico 3 se muestra el panorama comparativo de los resultados en la prueba TOIEC durante los años 2008 y 2011, en las regiones capacitadas por la UNED.

Se puede observar que en la banda A1 para el 2008 se ubicaban 72 docentes, para el 2011 se ve una disminución realmente significativa, bajando para el 2011 a solamente tres docentes. En la banda A2 se puede ver que en el 2008 se situaban 249 docentes, en tanto que, para el 2011 se observan solamente 33 en ella. Esto implica que 285 docentes se movilizaron de esta banda de usuario básico a otras bandas superiores.

En relación con la banda $\mathrm{B} 1$ de usuario independiente intermedio bajo, para el 2008 se ubicaban 279 docentes; para el año 2011, esta banda refleja 201. 


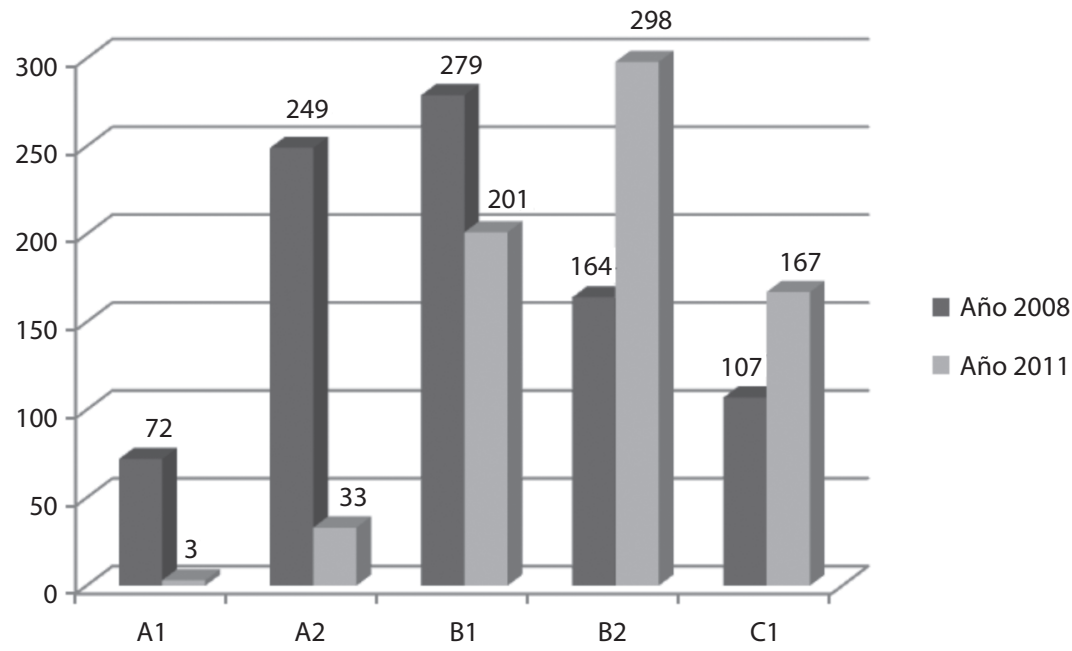

Gráfico 3. Distribución de docentes de inglés por banda en el año 2008 según resultados prueba TOEIC, regiones capacitadas por la UNED. Fuente: Archivos prueba TOEIC 2008-2011.

Como se indicó anteriormente en este documento, los/as docentes ubicados/as en las bandas B2 y $\mathrm{C} 1$ cuentan con el perfil profesional idóneo para la ejecución de las funciones dentro del Ministerio de Educación Pública; por esto, en el proyecto se concentraron esfuerzos para lograr que una gran mayoría de docentes que laboran en el MEP en los diferentes niveles y modalidades se ubicaran dichas categorías.

En la banda B2 de usuario independiente intermedio se observa, en el 2008, 164 docentes. Para el 2011, se incrementa en casi un $45 \%$, ya que se ubica un total de 298 docentes de Inglés en esta banda.
En la banda C1, de usuario proficiente, para el 2008 se ubicaban 107 docentes, mientras que para el 2011 mostró un incremento de un $60 \%$, es decir, 60 individuos más se ubican en esta banda, por lo tanto llegan a ubicarse un total de 167 docentes en $\mathrm{C} 1$.

\section{Conclusión}

En resumen, de los nueve cursos de dominio lingüístico ofrecidos como parte de este proyecto de capacitación, el porcentaje de aprobación fue bastante significativo, la mayoría de los cursos estuvo entre el $85 \%$ y el $95 \%$ de aprobación. 
En términos generales, en relación con el nivel lingüístico de los docentes, aquellas direcciones regionales de enseñanza a las cuales a la UNED le correspondió capacitar, evidencian una clara disminución de profesionales en las bandas más básicas de desempeño lingüístico y un aumento considerable de docentes ubicados en las bandas intermedias y avanzadas de dominio lingüístico, según los estándares del Marco Común Europeo de Referencia para la Lenguas Vivas. Se aprecia que, en su mayoría, los docentes aumentaron en general dos bandas como mínimo una vez finalizada la capacitación.

\section{Bibliografía}

Cámara Costarricense de Tecnologías de Información y Comunicación (2005, 30 de noviembre). Conclusiones del Primer Foro Nacional de Inglés [documento inédito], San José, Costa Rica.
Comisión Técnica Académica MEPCONARE-INGLÉS (2009). Informe 2008. San José, Costa Rica.

Comisión Técnica Académica MEPCONARE-INGLÉS (2010). Informe 2009. San José, Costa Rica.

Comisión Técnica Académica MEPCONARE-INGLÉS (2011). Informe 2010. San José, Costa Rica.

Comisión Técnica Académica MEPCONARE-INGLÉS (2012). Informe 2011. San José, Costa Rica.

Decreto 34 - 425 - MEP - COMEX del 11/03/2008. La Gaceta N. ${ }^{\circ}$ 61, viernes 28 de marzo del 2008. San José, Costa Rica.

MEP-CONARE (2008). Convenio Marco de Cooperación. San José, Costa Rica.

MEP-CONARE (2011). Convenio Específico entre el MEP y CONARE. San José, Costa Rica.

MEP, COMEX, MEIC, INA, CINDE \& Estrategia Siglo XXI (2007). Plan Nacional de Inglés (propuesta borrador). [Documento inédito]. San José, Costa Rica. 